\title{
Intolerance of uncertainty is associated with heightened responding in the prefrontal cortex during cue-signalled uncertainty of threat
}

\author{
Jayne Morriss ${ }^{1} \cdot$ Tiffany Bell $^{2}$. Nicolò Biagi ${ }^{1} \cdot$ Tom Johnstone $^{3} \cdot$ Carien M. van Reekum $^{1}$
}

Accepted: 9 July 2021 / Published online: 26 July 2021

(C) The Author(s) 2021

\begin{abstract}
Heightened responding to uncertain threat is considered a hallmark of anxiety disorder pathology. We sought to determine whether individual differences in self-reported intolerance of uncertainty (IU), a key transdiagnostic dimension in anxiety-related pathology, underlies differential recruitment of neural circuitry during cue-signalled uncertainty of threat $(n=42)$. In an instructed threat of shock task, cues signalled uncertain threat of shock (50\%) or certain safety from shock. Ratings of arousal and valence, skin conductance response (SCR), and functional magnetic resonance imaging were acquired. Overall, participants displayed greater ratings of arousal and negative valence, SCR, and amygdala activation to uncertain threat versus safe cues. IU was not associated with greater arousal ratings, SCR, or amygdala activation to uncertain threat versus safe cues. However, we found that high IU was associated with greater ratings of negative valence and greater activity in the medial prefrontal cortex and dorsomedial rostral prefrontal cortex to uncertain threat versus safe cues. These findings suggest that during cue-signalled uncertainty of threat, individuals high in IU rate uncertain threat as aversive and engage prefrontal cortical regions known to be involved in safety-signalling and conscious threat appraisal. Taken together, these findings highlight the potential of IU in modulating safety-signalling and conscious appraisal mechanisms in situations with cue-signalled uncertainty of threat, which may be relevant to models of anxiety-related pathology.
\end{abstract}

Keywords Instructed threat of shock $\cdot$ Intolerance of uncertainty $\cdot$ Medial prefrontal cortex $\cdot$ Rostral dorsomedial prefrontal cortex $\cdot$ fMRI

\section{Introduction}

In everyday life, we often experience uncertainty and will typically try to minimise or resolve it to reduce anxiety and stress (Brosschot, Verkuil, \& Thayer, 2016; Grupe \& Nitschke, 2013; Hirsh, Mar \& Peterson, 2012; Morriss, Gell, \& van Reekum, 2019b; Peters, McEwen, \& Friston, 2017). Individuals who score high in self-reported intolerance of uncertainty (IU) tend to find uncertainty particularly distressing (Carleton, 2016a, 2016b; Dugas, Buhr, \& Ladouceur, 2004;

Jayne Morriss

j.e.morriss@ reading.ac.uk

1 Centre for Integrative Neuroscience and Neurodynamics, School of Psychology and Clinical Language Sciences, University of Reading, Reading, UK

2 Department of Radiology, University of Calgary, Calgary, Canada

3 Department of Health and Medical Sciences, Swinburne University of Technology, Melbourne, Australia
Freeston, Rhéaume, Letarte, Dugas, \& Ladouceur, 1994). IU is considered a transdiagnostic dimension. High levels of IU are observed across many mental health disorders with an anxiety component, such as anxiety, depression, posttraumatic stress, and obsessive-compulsive disorder (Gentes \& Ruscio, 2011; McEvoy \& Mahoney, 2012). On this basis, there has been a surge in IU-related research in the field of anxiety during the past decade (McEvoy, Hyett, Shihata, Price, \& Strachan, 2019; Tanovic, Gee, \& Joormann, 2018).

Despite progress in understanding the aetiology of IU, gaps in the literature remain as to how IU modulates neural circuitry associated with the processing of cue-signalled uncertainty of threat (i.e., a cue signals whether a threat, such as an aversive stimulus, will occur or not) (Tanovic et al., 2018). Only a handful of studies to date have examined how IU is correlated with neural circuitry during the anticipation of cue-signalled uncertainty of threat (Morriss, Christakou, \& Van Reekum, 2015; Schienle, Köchel, Ebner, Reishofer, \& Schäfer, 2010; Simmons, Matthews, Paulus, \& Stein, 2008; Somerville et al., 2013). In tasks where participants have been instructed about 
the likelihoods of negative or neutral pictures (i.e., visual cues or countdowns signal the occurrence of a negative or neural picture), individuals high in IU, relative to low IU, have been shown to exhibit heightened amygdala and insula activity to cues signalling unpredictable negative pictures (Schienle et al., 2010; Shankman et al., 2014), and exaggerated amygdala activity to negative pictures following unpredictable countdowns (Somerville et al., 2013). Whilst previous work has provided a starting point for understanding how IU modulates neural circuitry to cue-signalled uncertainty of threat, further research is needed to assess the robustness and generalisability of IU-related effects. For example, previous fMRI research on the relationship between IU and cuesignalled uncertainty of threat has primarily used negative and neutral picture stimuli with a wide range of content. It is important to establish whether similar patterns of neural activation to cue-signalled uncertainty of threat would be observed for individuals with high IU in response to other stimuli commonly used to evoke anxious states, such as mild electric shock. Understanding how IU modulates neural circuitry in relation to cue-signalled uncertainty of threat will further clarify the role of IU in neurobiological (Grupe \& Nitschke, 2013; Peters et al., 2017) and clinical models of anticipation and uncertainty in anxiety (Carleton, 2016b; Shihata, McEvoy, Mullan, \& Carleton, 2016).

To assess the relationship between self-reported IU and anticipatory responding during cue-signalled uncertainty of threat, we measured event-related functional magnetic resonance imaging (fMRI), skin conductance response (SCR), and arousal and valence ratings while participants performed an instructed threat of shock task. To induce a sense of uncertain threat, a cue signalled whether a mild electric shock to the finger would occur (50\% of the time) (i.e., participants were told that they would sometimes receive a shock at the end of the cued trial). Trials were 9 seconds in length (1-second cue, 8 -second anticipatory period), to allow us to examine phasic and sustained threat/safety-related activity.

We hypothesized that, during the instructed threat of shock task, we would observe typical patterns of phasic and sustained activation in circuitry associated with the processing of threat and safety (Etkin, Egner, \& Kalisch, 2011; Levy \& Schiller, 2021; Mechias, Etkin, \& Kalisch, 2010; Morriss et al., 2019b; Tashjian, Zbozinek, \& Mobbs, 2021), i.e., (1) greater activation in the amygdala, putamen, caudate, insula, and rostral prefrontal cortex to threat versus safe trials, and (2) greater medial prefrontal cortex activity to safe versus threat trials. Moreover, we hypothesized that participants would display greater SCR to the threat versus safe trials and rate the threat trials as more negative and arousing than the safe trials.

Based on past research (Morriss et al., 2015; Schienle et al., 2010; Shankman et al., 2014; Simmons et al., 2008; Somerville et al., 2013), we hypothesised that higher IU would be associated with: (1) greater phasic and sustained activation in the amygdala and insula during threat, relative to safe trials, and (2) modulation of the medial prefrontal cortex during threat, relative to safe trials. Given the shortage of research on the relationship between IU and activation in the medial prefrontal cortex, we did not hypothesise a particular direction of effect. Lastly, we hypothesised that higher IU would be associated with greater SCR to the threat versus safe trials, as well as higher ratings of negativity and arousal to the threat versus safe trials.

We tested the specificity of the involvement of IU by comparing it with broader measures of anxiety (for discussion see (Morriss, Christakou, \& Van Reekum, 2016), in this case the Spielberger State-Trait Anxiety Inventory, Trait Version (STAIT) (Spielberger, Gorsuch, Lushene, Vagg, \& Jacobs, 1983).

\section{Methods}

\section{Participants}

Forty-two female volunteers were recruited from the local area through advertisements $(M$ age $=33 \mathrm{yr}, S D$ age $=7.33 \mathrm{yr} ; 31$ white Northern European, 6 white nonspecified region, 3 white Southern European, 1 multiethnic, 1 white Eastern European). All participants reported being right-handed, having normal or corrected-to-normal vision, being medicationfree, and having no prior history of brain injury. We did not collect information from participants regarding current or previous history of mental health diagnoses. We selected female participants, because the study was part of a larger programme of research examining the role of conspecifics (i.e., romantic partner, friend) in the processing of threat (Morriss, Bell, Johnstone, van Reekum, \& Hill, 2019a).

Participants provided written, informed consent and received a picture of their brain and $£ 15$ for their participation. The University of Reading's Research Ethics Committee approved the study protocol.

\section{Instructed threat of shock task}

Participants were required to passively view cues that represented either threat of shock or safety from shock. Only two cues were presented: an uncertain threat cue where there was $50 \%$ chance of receiving a shock, and a safety cue where there was $0 \%$ chance of receiving a shock. Each trial consisted of: a white cue (e.g., X, $\mathrm{O}, \mathrm{D}, \mathrm{T}$ ) presented on a black background (1 second), a white fixation anticipation cue presented on a black background ( 8 seconds), a small circle cue signalling the end of the trial (1 second), and a black blank screen (4-6 seconds) (Figure 1). In the uncertain threat condition, the shock was administered with the end cue $50 \%$ of the time. Participants completed 1 run of 36 trials (18 Threat, 18 Safe). To rule out any cue-specific 


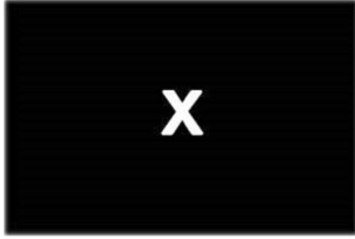

Threat Cue

$1 \mathrm{sec}$

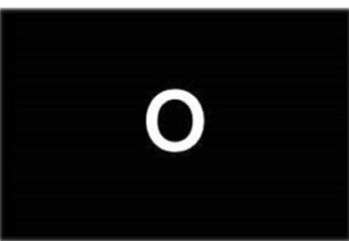

Safe Cue

$1 \mathrm{sec}$

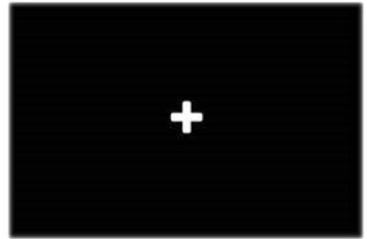

Anticipation

$8 \mathrm{sec}$

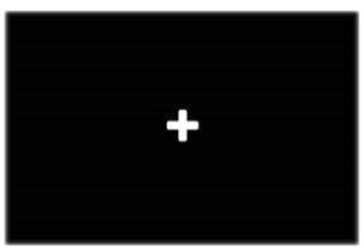

Anticipation

$8 \mathrm{sec}$

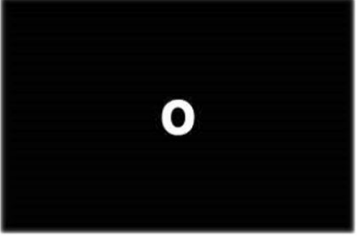

End Cue

$1 \mathrm{sec}$

(50\% shock

probability)

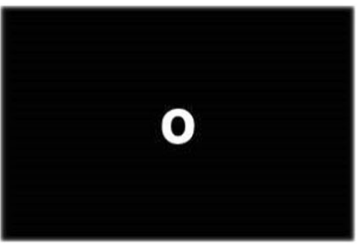

End Cue

No shock

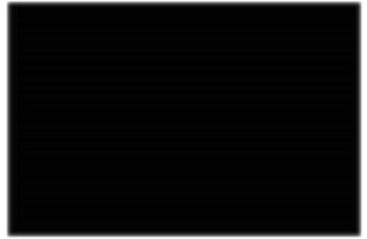

Rest

4-6 sec

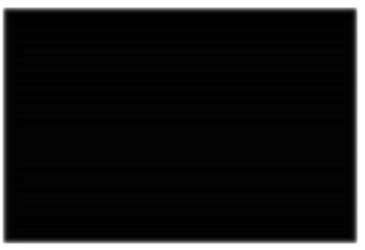

Rest

4-6 sec

Fig. 1 Image depicting instructed threat of shock task. Examples of threat (top row) and safe (bottom row) trial types. Participants were instructed on threat and safe contingencies before the start of the task

effects, half the participants received $\mathrm{X}$ and $\mathrm{O}$ cues, whilst the other half received $\mathrm{D}$ and $\mathrm{T}$ cues.

At the beginning of the experiment four practise trials (2 Threat, 2 Safe) were presented without shock on a computer screen. While the participants were viewing these practise trials, the experimenter explained that one of the cues would be associated with shock and another would not. Additionally, the experimenter explained that there would be a waiting period and that if the shock were to occur, it would happen at the same time as the small circle cue (Figure 1). The practise trials were given to allow the participants to experience the trial structure and timings. Before participants were placed in the scanner, they were instructed via a computer screen as to which cue would signal the possibility of a shock and which cue would signal no shock.

\section{Electric Shock}

The possibility of receiving an unpleasant electrical shock to the index and middle finger of the right hand was used to induce threat. Electric shocks were delivered via an ADInstruments ML856 PowerLab 26T Isolated Stimulator using MRI-safe MLT117F bipolar finger electrodes. Each participant's stimulation level was set by first exposing them to an electric stimulation of $1 \mathrm{~mA}(10$ pulses at $50 \mathrm{~Hz}$, with a pulse duration of $200 \mu \mathrm{s}$ ) and increasing the current in steps of $0.5 \mathrm{~mA}$, up to a maximum of $10 \mathrm{~mA}$. This continued until a suitable participant-specific threshold was found that was uncomfortable but not painful. This level was then used throughout the threat of shock task for that subject (electric stimulation level: $\mathrm{M}=2.21 \mathrm{~mA} ; \mathrm{SD}=1 \mathrm{~mA}$ ).

\section{Procedure}

Participants arrived at the laboratory and were informed of the experimental procedures. First, participants completed a consent form as an agreement to take part in the study. Second, participants completed questionnaires by pen and paper. Third, participants were taken to the MRI unit, where they were presented with example trials from the instructed threat of shock task and the shock procedure was carried out. Next, we presented instructions about the task contingencies, and then participants completed an instructed threat of shock task in the scanner whilst we concurrently recorded skin conductance. After scanning, participants rated the threat and safe cues from the instructed threat of shock task. Ratings were collected at the end of the scanning session rather than throughout the task, because participants had electrodes attached to both hands.

\section{Questionnaires}

To assess anxious disposition, we used Intolerance of Uncertainty (IU) (Freeston et al., 1994) and the Spielberger State-Trait Anxiety Inventory, Trait Version (STAI-T) (Spielberger et al., 1983). The IU scale comprises of 27 items on a 5-point Likert scale (possible range: 20-135) and captures aversion to uncertainty. The STAI-T comprises of 20 items on a 4-point Likert scale (possible range: 20-80) and captures trait anxiety. Both anxiety measures were normally distributed, showed good internal reliability and displayed similar ranges of scores to that of community samples (IU: $M=66.07 ; S D=$ $17.03 ;$ range $=34-102 ; \alpha=0.93 ;$ STAI-T; $M=40.92 ; S D=$ 
10.31; range $=25-61 ; \alpha=0.91)(J u l i a n, 2011 ;$ Khawaja \& Yu, 2010). Importantly, at least half of the sample met STAI-T $(>40)$ and IU (>60) scores that were comparable with clinical samples (Julian, 2011; Khawaja \& Yu, 2010).

\section{Ratings}

Participants rated the valence and arousal of the threat and safe cues using 9-point Likert scales ranging from 1 (Valence: negative; Arousal: calm) to 9 (Valence: positive; Arousal: excited). One participant did not complete the ratings, leaving 41 participants with ratings data.

\section{Skin conductance acquisition and reduction}

Identical to previous work (Morriss et al., 2015), electrodermal recordings were obtained using $\mathrm{AD}$ Instruments (AD Instruments Ltd, Chalgrove, Oxfordshire) hardware and software. An ML138 Bio Amp connected to an ML870 PowerLab Unit Model 8/30 amplified the electrodermal signal, which was digitized through a 16-bit A/D converter at 1,000 Hz. Electrodermal activity was measured during the scanning session with MRI-safe MLT117F Ag/AgCl bipolar finger electrodes filled with $\mathrm{NaCl}$ electrolyte paste (Mansfield R \& D, St. Albans, VT) that were attached to the distal phalanges of the index and middle fingers of the left hand. A constant voltage of $22 \mathrm{mVrms}$ at $75 \mathrm{~Hz}$ was passed through the electrodes, which were connected to a ML116 GSR Amp.

Skin conductance responses (SCR) were scored when there was an increase of skin conductance level exceeding 0.03 microSiemens. The amplitude of each SCR was scored as the difference between the baseline (1-second average pre cue onset) and the maximum deflection (0.5-9-second post cue onset). Trials with no discernible SCRs were scored as zero. SCR magnitudes were calculated by averaging SCR values for each condition (Threat, Safe). Due to recording errors, 2 participants did not have SCR data, leaving 40 participants with SCR data.

\section{Ratings and SCR analysis}

We conducted a two Condition (Threat, Safe) x IU ANCOVA for arousal ratings, valence ratings, and SCR to the cues, where IU was entered as a continuous predictor variable. Any interaction with IU was followed up with pairwise comparisons of the means between the conditions for IU estimated at the specific values of + or -1 SD of mean IU. This type of analysis with IU has been previously published elsewhere (Morriss et al., 2015, 2016).

We performed hierarchical regression analyses on the rating/SCR difference score measures that showed an effect with IU. This analysis served to assess IU-specific effects over and above shared variance with trait anxiety (STAI-T). We entered STAI-T in the first step and then IU in the second step.

\section{MRI}

Participants were scanned with a 3 T Siemens Trio using a 12 channel head coil (Siemens Inc., Erlangen, Germany). T2*weighted gradient-echo, echo planar imaging (EPI) functional scans were acquired for the threat of shock task consisting of 281 volumes $\left(\mathrm{TR}=2,000 \mathrm{~ms}, \mathrm{TE}=30 \mathrm{~ms}\right.$, flip angle $=90^{\circ}$, FOV $=192 \times 192 \mathrm{~mm}, 3-\times 3-\mathrm{mm}$ voxels, slice thickness $3 \mathrm{~mm}$ with an interslice gap of $1 \mathrm{~mm}, 30$ axial slices, interleaved acquisition).

Following completion of the functional scan, structural and fieldmap scans were acquired, which comprised of a highresolution, T1-weighted anatomical scan (MP-RAGE, TR = $2020 \mathrm{~ms}, \mathrm{TE}=2.52 \mathrm{~ms}$, flip angle $=90^{\circ}, \mathrm{FOV}=256 \times 256$ $\mathrm{mm}, 1-\mathrm{x} 1-\mathrm{x} 1-\mathrm{mm}$ voxels, slice thickness $1 \mathrm{~mm}$, sagittal slices) and fieldmap (TR $=488 \mathrm{~ms}$, TE $1=4.98 \mathrm{~ms}$, TE $2=$ $7.38 \mathrm{~ms}$, flip angle $=60^{\circ}, \mathrm{FOV}=256 \times 256 \mathrm{~mm}$, slice thickness $4 \mathrm{~mm}$ with an interslice gap of $4 \mathrm{~mm}, 30$ axial slices).

\section{fMRI analysis}

FMRI analyses were carried out in Feat version 5.98 as part of FSL (FMRIB's Software Library, www.fmrib.ox.ac.uk/fsl). Brains were extracted from their respective T1 images by using the FSL Brain Extraction Tool (BET) (Smith, 2002). Distortion, slice timing and motion correction were applied to all extracted EPI volumes using FUGUE and MCFLIRT tools. Gaussian smoothing (FWHM $5 \mathrm{~mm}$ ) and a 50-second high-pass temporal filter were applied.

A first-level GLM analysis was carried out for each functional scan. Separate regressors were specified for the experimental conditions of primary interest (Threat/Safety Cues) by convolving a binary boxcar function with an ideal haemodynamic response (HR), which corresponded to the length of each cue (1 second) or the entire trial period ( 9 seconds). Regressors for the end of trial period with and without shock and six motion parameters ( 3 rotation and 3 translation) were included to model out brain activity that was unrelated to the conditions of interest.

In two separate general linear models, we defined two main effect contrasts to reveal phasic and sustained threat/ safety related activity: (1) Threat vs. Safety across the 1second cue period, and (2) Threat vs. Safety across the whole 9-second trial period. All contrasts were normalized and registered to MNI standard space using FLIRT (Jenkinson, Bannister, Brady, \& Smith, 2002). Secondlevel GLM analysis consisted of regressors for the group mean and demeaned IU scores using FSL's OLS procedure. Whole-brain analysis was carried out with parametric statistics using cluster thresholding with a $z=2.3$ and 
a corrected $p<0.05$ (for fMRI results from nonparametric permutation tests, see Supplementary Material).

We performed hierarchical regression analyses on the resulting significant clusters that showed an association with IU to test for specificity of IU over STAI-T. We extracted \% BOLD signal change difference scores from the relevant clusters and correlated these with the anxiety measures to test for IU-specific effects, by using STAI-T in the first step and then STAI-T and IU in the second step of the hierarchical regression models.

\section{Results}

\section{Ratings}

Participants rated the threat cues as more negative $(M=4.78$, $S D=1.77)$ and more arousing $(M=5.78, S D=1.68)$ than the safe cue $(M=6.78, S D=1.56$ for valence and $M=2.90, S D=$ 2.11 for arousal respectively) [Condition (Valence): $F(1,39)=$

A

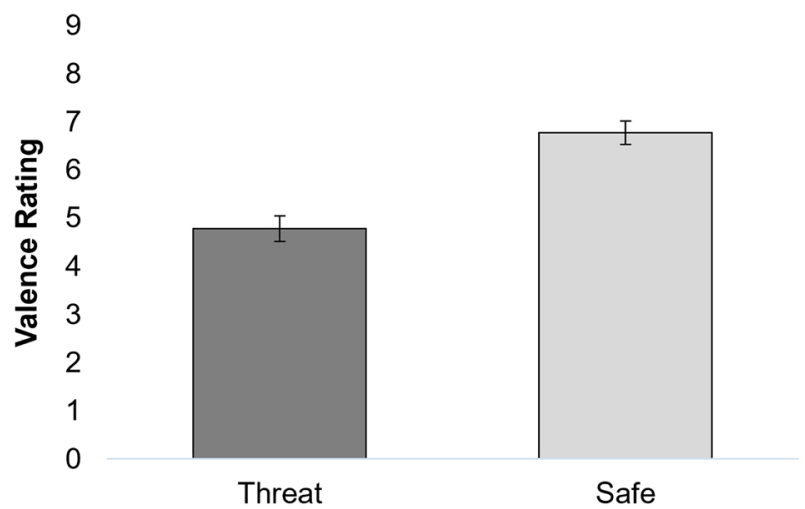

C

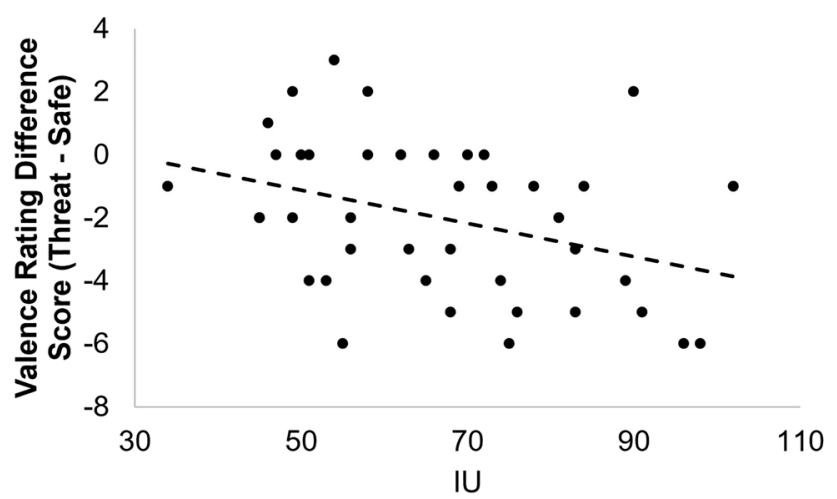

Fig. 2 Bar graphs depicting valence and arousal ratings for threat and safe stimuli (a \& b). Higher IU was significantly associated with rating the threat stimulus as more negative than the safe stimulus (c). IU was not
29.127, $p<0.001$; Condition (Arousal): $F(1,39)=47.095, p<$ 0.001 ; Figures $2 \mathrm{~A} \& \mathrm{~B}]$. Higher IU was associated with significantly more negative ratings of the threat cues compared to the safe cues, $p<0.001$ [Condition (Valence) x IU interaction: $F(1,39)=5.764, p=0.021$; Figure $2 \mathrm{C}]$. IU was not significantly related to the arousal ratings of the threat and safe cues [Condition (Arousal) x IU interaction: $F(1,39)=0.866, p=$ 0.358; Figure 2D].

For the valence rating difference score (Threat cue - Safe cue), STAI-T made no significant contribution to the model at the first step $\left[R^{2}=0.044, F=1.808\right]$, whilst adding IU improved the hierarchal model at trend in the second step $\left[\Delta R^{2}=\right.$ $0.086, F(1,38)=3.746, p=0.06]$.

\section{SCR}

SCR was greater to threat $(M=0.29, S D=0.11)$ versus safe $(M=0.16, S D=0.11)$ trials [Condition: $F(1,38)=$ 43.815, $p<0.001]$. Higher IU was related to greater

B

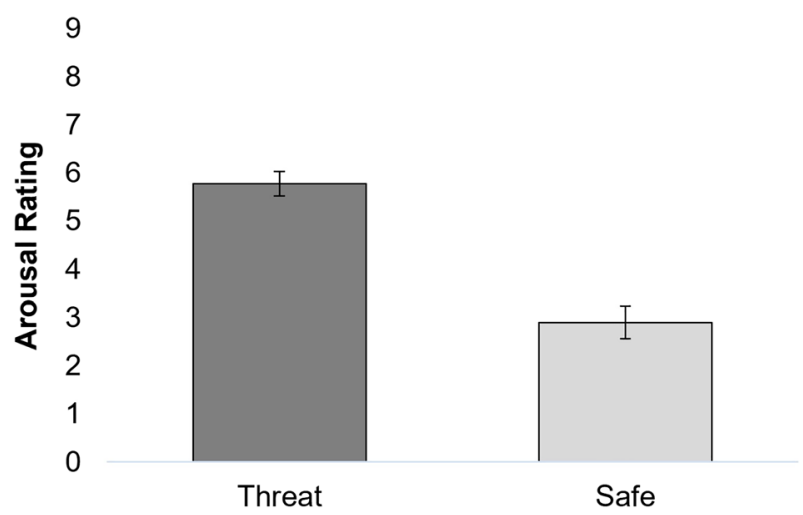

D

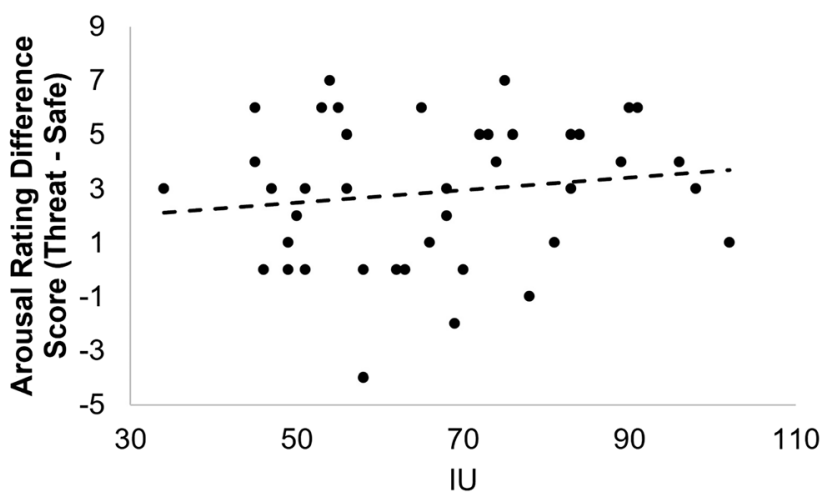

significantly related to arousal rating difference scores between threat and safe stimuli (d). Valence, $1=$ negative and $9=$ positive; Arousal $1=$ calm, $9=$ excited. Bars represent standard error 
SCR to threat versus safe trials; however, this relationship was not significant [Condition x IU: $F(1,38)=$ $3.059, p=0.088]$.

\section{fMRI}

For all participants, threat versus safe cues induced greater activation in the bilateral amygdala, insula, frontal operculum, pre- and postcentral gyrus, paracingulate, cingulate, supramarginal gyrus, and middle frontal gyrus (for full list of brain regions see Table $1 \&$ Figure 3). During threat versus safe trial periods, activations were observed in the bilateral insula, caudate, putamen, orbital frontal cortex, supramarginal gyrus, middle frontal gyrus, thalamus, and brain stem (for full list of brain regions see Table $1 \&$ Figure 4), The reverse contrast, safe versus threat trial period, revealed greater activation in the bilateral hippocampus, medial cortex, superior frontal and middle frontal gyri, and precuneus (for full list of brain regions see Table $1 \&$ Figure 4).

For threat versus safe cues, high IU was associated with greater activation in the medial prefrontal cortex and rostral dorsomedial prefrontal cortex (split into two clusters, see Table $1 \&$ Figure 5). No significant IU-related effects were observed for the safe versus threat contrast for the cue period. In addition, no significant IU-related effects were found for the contrasts from the trial period.

For the medial prefrontal cortex cluster (Threat cue - Safe cue), STAI-T made a significant contribution to the model at the first step $\left[R^{2}=0.220, F(1,40)=11.301, p=0.002\right]$, and adding IU in the second step improved the model significantly $\left[\Delta R^{2}=0.167, F(1,39)=10.610, p=0.002\right]$. Similarly, for the rostral dorsomedial prefrontal cortex cluster (Threat cue Safe cue), STAI-T made a significant contribution to the model at the first step $\left[R^{2}=0.218, F(1,40)=11.171, p=0.002\right]$, whilst adding IU improved the model significantly in the second step $\left[\Delta R^{2}=0.163, F(1,39)=10.287, p=0.003\right]$.

\section{Discussion}

In the following experiment, self-reported IU was not found to be associated with amygdala or insula activity during cuesignalled uncertainty of threat. However, higher IU was specifically associated with greater recruitment of the medial prefrontal cortex and dorsomedial rostral prefrontal cortex to cues that signalled uncertainty of threat of shock versus safety from shock, over STAI-T. Furthermore, IU-related effects were specific to the cue (phasic); we did not observe IU modulation of neural activity during the entire trial period (sustained). These results highlight the potential of IU-based modulation of mechanisms related to safety-signalling and conscious threat appraisal in anxiety disorder pathology.
In general, we found that participants recruited typical regions associated with instructed threat of shock tasks (Etkin et al., 2011; Grupe \& Nitschke, 2013; Levy \& Schiller, 2021; Mechias et al., 2010; Morriss et al., 2019b; Tashjian et al., 2021). Participants recruited greater amygdala to threat versus safe cues (phasic), as well as greater putamen, caudate, and insula during threat versus safe trial periods (cue + anticipation window). Moreover, participants recruited greater medial prefrontal cortex during safe versus threat periods. As expected, greater SCR was observed to the threat versus safe trials. Furthermore, participants rated threat cues as negative and moderately arousing, and safe cues as moderately positive and low in arousal.

We did not observe higher IU to differentially engage the amygdala or insula (across the cue or entire trial period) or display greater SCR to cues that signalled uncertainty of threat of shock versus safety from shock. Whilst this is at odds with previous research (Morriss et al., 2015; Schienle et al., 2010; Shankman et al., 2014; Simmons et al., 2008; Somerville et al., 2013), there may be an explanation for these results. In the current study, the dominant source of uncertainty was outcome uncertainty of threat (i.e., if a threat (shock) would occur or not), whereas in previous studies there have been multiple sources of uncertainty of threat (i.e., if, when, and what type of negative picture would be displayed) (Schienle et al., 2010; Shankman et al., 2014; Simmons et al., 2008; Somerville et al., 2013). Many parameters of uncertainty in combination may be perceived as more threatening and arousing in general, but particularly in individuals who score higher in IU. Therefore, in a context where different parameters of uncertainty are combined, threat cues are more likely to engage circuitry, such as the amygdala and insula, and arousal-based physiology, such as SCR, to alert the individual to this particular situation of threat. Our results and previous research need to be further explored and replicated to fully understand how IU modulates neural circuitry under different parameters of uncertainty of threat - ideally in a single study where different parameters of uncertainty of threat are isolated (i.e., if, when, and what; instructed vs. uninstructed) (Bennett, Dickmann, \& Larson, 2018; Davies \& Craske, 2015; Mertens \& Morriss, 2021; Morriss, Bennett, \& Larson, 2021; Morriss, Biagi, \& Dodd, 2020).

The medial prefrontal cortex has been implicated in threat regulation and safety-signalling (Etkin et al., 2011; Milad \& Quirk, 2012; Tashjian et al., 2021). In this task, higher IU was associated with greater recruitment of the medial prefrontal cortex during threat versus safe cues. Given that the contingencies were instructed, this finding may reflect attempts to update the value of the threat cue as less threatening or safe in individuals with high IU. The modulation of activity in this area by IU is in line with prior work showing high IU individuals to recruit more medial prefrontal cortex during the extinction of threat versus safe cues, where the values of cues change 
Table 1 Regional activation patterns in response to stimuli presented in the threat of shock task

\begin{tabular}{|c|c|c|c|c|c|c|c|}
\hline \multirow[t]{2}{*}{ Task } & \multirow[t]{2}{*}{ Brain region } & \multirow[t]{2}{*}{ BA } & \multirow{2}{*}{$\begin{array}{l}\text { Voxels } \\
\left(\mathrm{mm}^{3}\right)\end{array}$} & \multirow[t]{2}{*}{ Max Z } & \multicolumn{3}{|c|}{ Location of $\max \mathrm{Z}$} \\
\hline & & & & & $\mathrm{x}$ & $\mathrm{y}$ & $\mathrm{z}$ \\
\hline \multicolumn{8}{|c|}{ Threat of Shock (Cue Period) } \\
\hline Threat $>$ Safe & $\begin{array}{l}\text { L amygdala, insula cortex, frontal operculum cortex, inferior } \\
\text { frontal gyrus, frontal pole, middle frontal gyrus, } \\
\text { supramarginal gyrus, postcentral gyrus, precentral gyrus }\end{array}$ & $44 / 10 / 6 / 8 / 9 / 40 / 1 / 3$ & 5722 & 4.35 & -36 & 14 & 0 \\
\hline Threat $>$ Safe & $\begin{array}{l}\mathrm{R} \text { amygdala, insula cortex, frontal operculum cortex, inferior } \\
\text { frontal gyrus, frontal pole, middle frontal gyrus }\end{array}$ & $44 / 6 / 8 / 9 / 40$ & 4791 & 4.43 & 40 & 12 & 16 \\
\hline Threat $>$ Safe & $\begin{array}{l}\text { R parietal operculum cortex, middle temporal gyrus, } \\
\text { supramarginal gyrus }\end{array}$ & $21 / 40$ & 3144 & 5.17 & 52 & -42 & 6 \\
\hline Threat $>$ Safe & Cingulate gyrus, paracingulate gyrus & $24 / 32$ & 1344 & 4.18 & -8 & 2 & 40 \\
\hline Threat $>$ Safe & Superior frontal gyrus & $6 / 8$ & 313 & 4.01 & -6 & 40 & 54 \\
\hline Threat $>$ Safe & Posterior cingulate gyrus & 23 & 308 & 4.1 & 16 & -18 & 38 \\
\hline Threat $>$ Safe & R Superior frontal gyrus, precentral gyrus & $6 / 8 / 4$ & 282 & 3.58 & 16 & 0 & 66 \\
\hline Threat $>$ Safe $x$ IU & $\begin{array}{l}\text { paracingulate gyrus, frontal medial cortex, frontal pole, } \\
\text { superior frontal gyrus (medial prefrontal cortex) }\end{array}$ & $32 / 10 / 9$ & 894 & 4 & 2 & 58 & 30 \\
\hline Threat $>$ Safe $x$ IU & $\begin{array}{l}\text { paracingulate gyrus, Frontal pole (rostral dorsomedial } \\
\text { prefrontal cortex) }\end{array}$ & $32 / 8$ & 258 & 3.12 & 14 & 46 & 32 \\
\hline \multicolumn{8}{|c|}{ Threat of Shock (Cue and Anticipation Period) } \\
\hline Threat $>$ Safe & $\begin{array}{l}\text { L insula cortex, frontal operculum cortex, inferior frontal } \\
\text { gyrus, frontal pole, orobtial frontal cortex, putamen, } \\
\text { caudate }\end{array}$ & $47 / 45 / 44$ & 3184 & 5.94 & -32 & 22 & 12 \\
\hline Threat $>$ Safe & $\begin{array}{l}\mathrm{R} \text { insula cortex, frontal operculum cortex, inferior frontal } \\
\text { gyrus, frontal pole, orobtial frontal cortex, putamen, } \\
\text { caudate }\end{array}$ & $47 / 45 / 44$ & 2979 & 5.24 & 34 & 24 & -8 \\
\hline Threat $>$ Safe & Cingulate, paracingulate, juxtapositional lobule cortex & $24 / 32 / 4 / 6$ & 2447 & 4.67 & 2 & 10 & 62 \\
\hline Threat $>$ Safe & R supramarginal gyrus, parietal operculum cortex & 40 & 1421 & 4.77 & 56 & -42 & 40 \\
\hline Threat $>$ Safe & L supramarginal gyrus, parietal operculum cortex & 40 & 1359 & 4.66 & -56 & -24 & 18 \\
\hline Threat $>$ Safe & Brain stem, thalamus & & 1292 & 4.44 & 2 & -16 & -10 \\
\hline Threat $>$ Safe & Cerebellum & & 540 & 3.85 & 2 & -50 & -24 \\
\hline Threat $>$ Safe & Occipital pole & 17 & 393 & 4.45 & 34 & -98 & 0 \\
\hline Threat $>$ Safe & Precentral gyrus, middle frontal gyrus & $4 / 6 / 8$ & 338 & 3.96 & 42 & 4 & 52 \\
\hline Safe $>$ Threat & $\begin{array}{l}\text { posterior cingulate, precuneus cortex, occiptial pole, lingual } \\
\text { gyrus, L hippocampus, R hippocampus }\end{array}$ & 23/7/17/18/19 & 18491 & 6.11 & 10 & -56 & 18 \\
\hline Safe $>$ Threat & $\begin{array}{l}\text { Subcallosal cortex, paracingulate gyrus, frontal medial } \\
\text { cortex, frontal pole, }\end{array}$ & $12 / 25 / 32 / 10$ & 4671 & 6.44 & 4 & 48 & -8 \\
\hline Safe $>$ Threat & L superior frontal gyrus, middle frontal gyrus & $6 / 8 / 9$ & 924 & 4.27 & -22 & 22 & 38 \\
\hline Safe $>$ Threat & R superior frontal gyrus, middle frontal gyrus & $6 / 8 / 9$ & 727 & 4.74 & 24 & 32 & 44 \\
\hline Safe $>$ Threat & L superior temporal gyrus, middle temporal gyrus & $22 / 38$ & 668 & 4.48 & -64 & -8 & -14 \\
\hline
\end{tabular}

Note: Corrected cluster for multiple comparisons at $p<0.05$. BA $=$ Brodmann Areas. Location of cluster's maximum Z are in MNI space. $\mathrm{R}=$ right; $\mathrm{L}=$ left.

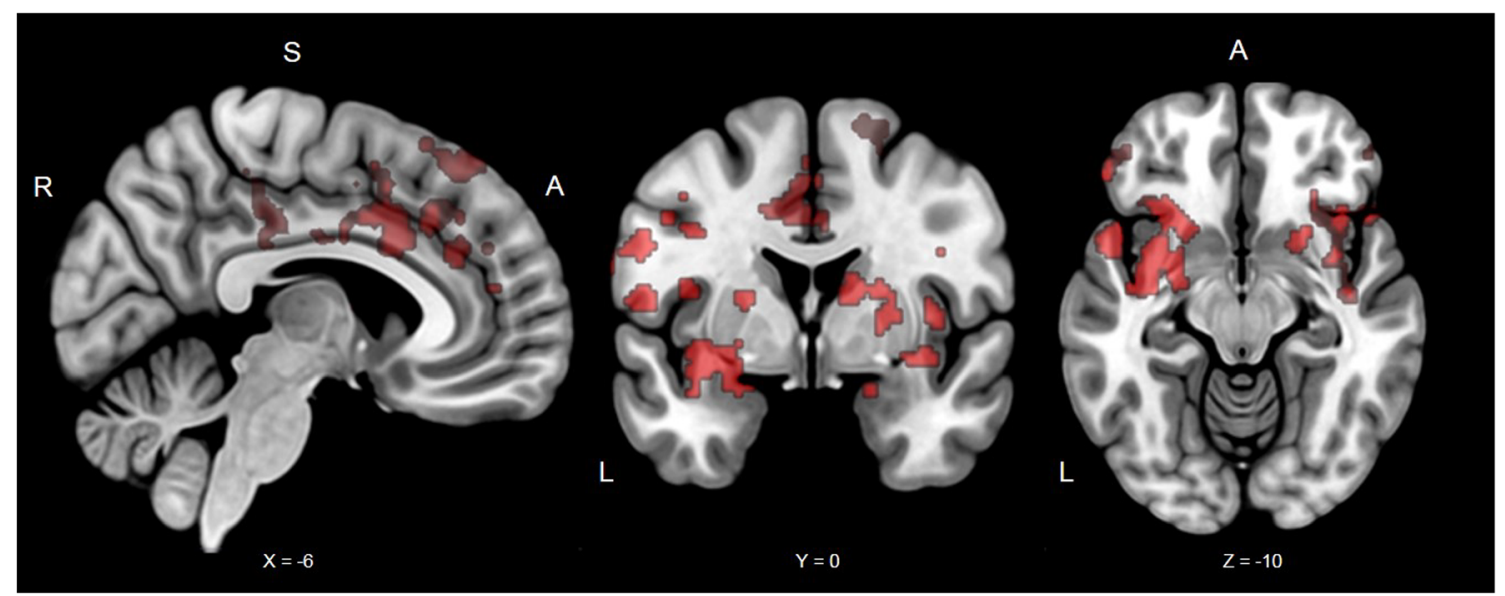

Fig. 3 Significant clusters from the instructed threat of shock task for all participants during the cue period. Typical regions activated during threat and safety were observed. The red clusters are from the Threat > Safe contrast. Coordinates in MNI space; R, right; S, superior; A, Anterior 


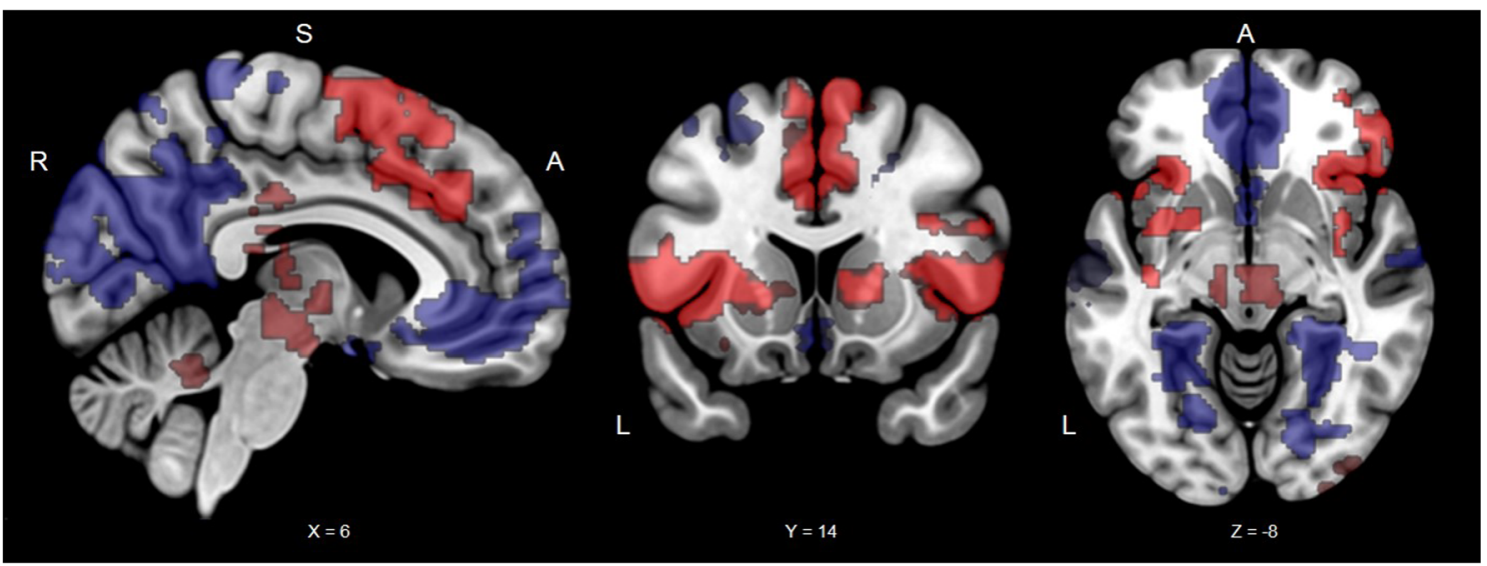

Fig. 4 Significant clusters from the instructed threat of shock task for all participants during the cue and anticipation period. Typical regions activated during threat and safety were observed. The red clusters are from the Threat $>$ Safe contrast and the blue clusters are from the Safe $>$ Threat contrast. Coordinates in MNI space; R, right; S, superior; A, Anterior

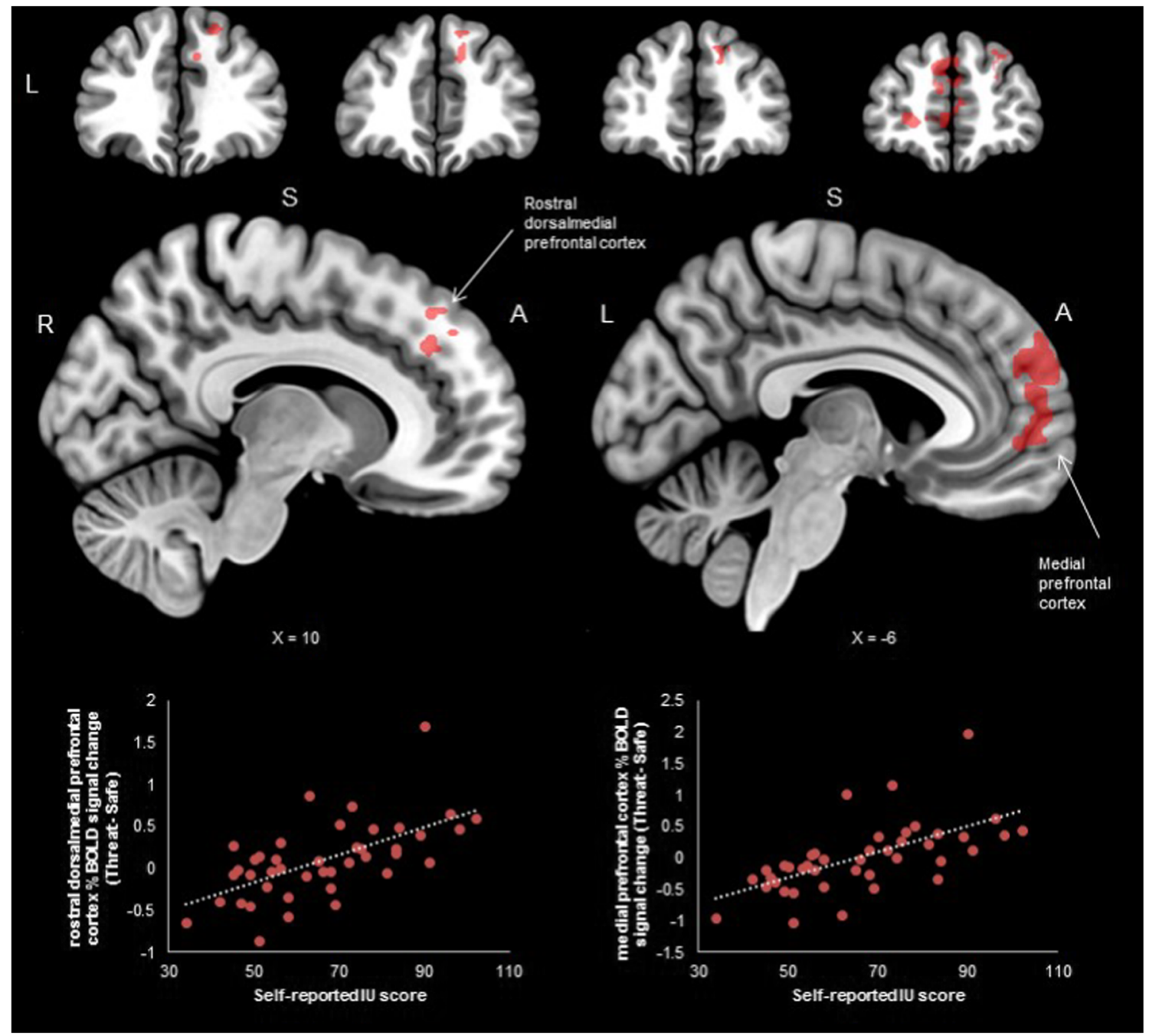

Fig. 5. Significant clusters from the instructed threat of shock task during the cue period by individual differences in self-reported intolerance of uncertainty (IU). For threat vs. safe cues, high IU was associated with greater activation in the rostral dorsomedial prefrontal cortex and medial prefrontal cortex (see bottom of figure for correlations). Such prefrontal regions are thought to be related to safety-signalling and conscious threat appraisal. Coordinates in MNI space; R, right; S, superior; A, Anterior 
from threat to safe (Morriss et al., 2015). Higher IU also was associated with greater dorsomedial rostral prefrontal cortex to cues signalling uncertainty of threat of shock versus safety from shock. In a recent meta-analysis, the dorsomedial rostral prefrontal cortex has been suggested to underlie conscious threat appraisal during instructed threat conditioning (Mechias et al., 2010) and generally involved in estimating threat (Grupe \& Nitschke, 2013). Therefore, in the context of instructed threat of shock, greater engagement of the dorsomedial rostral prefrontal cortex may reflect conscious threat appraisal in individuals high in IU. Alongside these neural findings, we also observed individuals with high IU, relative to low IU, to rate the threat cue as more aversive. The IU-related effects for the ratings provide further evidence that individuals with higher IU found the threat cue aversive, despite being instructed about threat and safe contingencies.

Although unexpected, IU-related effects in the medial prefrontal and dorsomedial rostral prefrontal cortex were only observed for the cue period (and not the trial period) during the instructed threat of shock task. Tentatively, these findings suggest that individuals with high IU may find the cue period to be particularly salient, as this is the point at which estimates of threat and safety can be computed. Perhaps, in the context of instructed threat of shock, individuals with high IU, relative to low IU are motivated to identify the cue to estimate threat and safety based on previous contingency instruction. However, once the cue is identified and the "risk" is known, individuals with high IU show similar anticipation of the outcome to that of individuals with low IU.

In neurobiological models of uncertainty and anticipation (Grupe \& Nitschke, 2013), the medial and dorsomedial rostral prefrontal cortex are thought to be involved in estimating threat and uncertainty, and signalling safety respectively. Notably, evidence-based therapies, such as Cognitive Behavioural Therapy (CBT), are designed to improve flexibility in estimates of threat, safety, and uncertainty in anxious populations (Clark \& Beck, 2011; Saklovskis, 1996). Therefore, the IU-based modulation of neurocircuitry implicated in estimates of threat, safety, and uncertainty within this study demonstrates promise for IU as a fundamental dimension in neurobiological models of uncertainty and anticipation (Grupe \& Nitschke, 2013; Peters et al., 2017), as well as a potentially useful transdiagnostic treatment target in evidencebased therapies, such as CBT (Dugas et al., 2003; Oglesby, Allan, \& Schmidt, 2017; Robichaud \& Dugas, 2006).

The study did have a few shortcomings and limitations. First, while the study did include practise trials that provided participants with experience of the temporal structure of the task (i.e., anticipation time before shock), the study did not use an explicit countdown to the shock (Shankman et al., 2014; Somerville et al., 2013), which could have been beneficial to remove any additional sources of temporal uncertainty of threat. Second, the generality of these IU-related findings should be tested in future studies using aversive stimuli other than shocks and with different reinforcement rates of cuesignalled uncertainty of threat (Chin, Nelson, Jackson, \& Hajcak, 2016). Lastly, the sample was relatively small and contained only female participants. Future studies should aim to recruit larger samples from more diverse community or clinical samples (Hiser, Schneider, \& Koenigs, 2021).

Taken together, these results suggest that, during cuesignalled uncertainty of threat, IU is specifically related over STAI-T to activation in prefrontal cortical regions. These preliminary findings highlight the potential of IU in altering safety-signalling and conscious threat appraisal mechanisms in anxiety disorder pathololgy (Brosschot et al., 2016; Carleton, 2016a, 2016b; Grupe \& Nitschke, 2013; Hirsh et al., 2012). Further research is needed to explore the generalisability of IU-related effects during cue-signalled uncertainty of threat, and how individual differences in IU modulate different parameters of uncertainty of threat (i.e., if, when, and what, as well as instructed vs. uninstructed)

Supplementary Information The online version contains supplementary material available at https://doi.org/10.3758/s13415-021-00932-7.

Acknowledgments This research was supported by funding from the University of Reading, by a NARSAD Young Investigator Grant from the Brain \& Behavior Research Foundation (27567) and an ESRC New Investigator Grant (ES/R01145/1) awarded to Jayne Morriss. The authors thank the participants who took part in this study and members of the CINN for their advice.

Data availability Data from this project are available on the Open Science Framework: https://osf.io/d28vy/.

Open Access This article is licensed under a Creative Commons Attribution 4.0 International License, which permits use, sharing, adaptation, distribution and reproduction in any medium or format, as long as you give appropriate credit to the original author(s) and the source, provide a link to the Creative Commons licence, and indicate if changes were made. The images or other third party material in this article are included in the article's Creative Commons licence, unless indicated otherwise in a credit line to the material. If material is not included in the article's Creative Commons licence and your intended use is not permitted by statutory regulation or exceeds the permitted use, you will need to obtain permission directly from the copyright holder. To view a copy of this licence, visit http://creativecommons.org/licenses/by/4.0/.

\section{References}

Bennett, K. P., Dickmann, J. S., \& Larson, C. L. (2018). If or when? Uncertainty's role in anxious anticipation. Psychophysiology, 55(7), e13066.

Brosschot, J. F., Verkuil, B., \& Thayer, J. F. (2016) The default response to uncertainty and the importance of perceived safety in anxiety and stress: An evolution-theoretical perspective. Journal of Anxiety Disorder, 41, 22-34 
Carleton, R. N. (2016a). Fear of the unknown: One fear to rule them all? Journal of Anxiety Disorders, 41, 5-21.

Carleton, R. N. (2016b). Into the unknown: A review and synthesis of contemporary models involving uncertainty. Journal of Anxiety Disorders, 39, 30-43.

Chin, B., Nelson, B. D., Jackson, F., \& Hajcak, G. (2016). Intolerance of uncertainty and startle potentiation in relation to different threat reinforcement rates. International Journal of Psychophysiology, 99, 79-84.

Clark, D. A., \& Beck, A. T. (2011). Cognitive therapy of anxiety disorders: Science and practice: Guilford Press.

Davies, C. D., \& Craske, M. G. (2015). Psychophysiological responses to unpredictable threat: Effects of cue and temporal unpredictability. Emotion, 15(2), 195.

Dugas, M. J., Buhr, K., \& Ladouceur, R. (2004). The Role of Intolerance of Uncertainty in Etiology and Maintenance of Generalized Anxiety Disorder. In: R. G. Heimberg, C. L. Turk, \& D. S. Mennin (Eds.), Generalized anxiety disorder: advances in research and practice (pp. 143-163). Guilford Press.

Dugas, M. J., Ladouceur, R., Léger, E., Freeston, M. H., Langolis, F., Provencher, M. D., \& Boisvert, J.-M. (2003). Group cognitivebehavioral therapy for generalized anxiety disorder: treatment outcome and long-term follow-up. Journal of Consulting and Clinical Psychology, 71(4), 821.

Etkin, A., Egner, T., \& Kalisch, R. (2011). Emotional processing in anterior cingulate and medial prefrontal cortex. Trends in Cognitive Sciences, 15(2), 85-93.

Freeston, M. H., Rhéaume, J., Letarte, H., Dugas, M. J., \& Ladouceur, R. (1994). Why do people worry? Personality and Individual Differences, 17(6), 791-802.

Gentes, E. L. \& Ruscio, A. M. (2011) A meta-analysis of the relation of intolerance of uncertainty to symptoms of generalized anxiety disorder major depressive disorder and obsessive-compulsive disorder. Clinical Psychology Review, 31(6), 923-933.

Grupe, D. W., \& Nitschke, J. B. (2013). Uncertainty and anticipation in anxiety: an integrated neurobiological and psychological perspective. Nature Reviews Neuroscience, 14(7), 488-501.

Hirsh, J. B., Mar, R. A., \& Peterson, J. B. (2012). Psychological entropy: a framework for understanding uncertainty-related anxiety. Psychological Review, 119(2), 304

Hiser, J., Schneider, B., \& Koenigs, M. (2021). Uncertainty potentiates neural and cardiac responses to visual stimuli in anxiety disorders. Biological Psychiatry: Cognitive Neuroscience and Neuroimaging, 6(7), 725-734.

Jenkinson, M., Bannister, P., Brady, M., \& Smith, S. (2002). Improved optimization for the robust and accurate linear registration and motion correction of brain images. Neuroimage, 17(2), 825-841.

Julian, L. (2011). Measures of anxiety: State-Trait Anxiety Inventory (STAI), Beck Anxiety Inventory (BAI), and Hospital Anxiety and Depression Scale-Anxiety (HADS-A). Arthritis Care \& Research, 63, S467-S472.

Khawaja, N. G., \& Yu, L. N. H. (2010). A comparison of the 27-item and 12-item intolerance of uncertainty scales. Clinical Psychologist, 14(3), 97-106.

Levy, I., \& Schiller, D. (2021). Neural computations of threat. Trends in Cognitive Sciences, 25(2), 151-171.

McEvoy, P. M., Hyett, M. P., Shihata, S., Price, J. E., \& Strachan, L. (2019). The impact of methodological and measurement factors on transdiagnostic associations with intolerance of uncertainty: A metaanalysis. Clinical Psychology Review, 73, 101778. doi:https://doi. org/10.1016/j.cpr.2019.101778

McEvoy, P. M. \& Mahoney, A. E. J. (2012) To Be Sure To Be Sure: Intolerance of Uncertainty Mediates Symptoms of Various Anxiety Disorders and Depression. Behavior Therapy, 43(3), 533-545.
Mechias, M. L., Etkin, A., \& Kalisch, R. (2010). A meta-analysis of instructed fear studies: implications for conscious appraisal of threat. Neuroimage, 49(2), 1760-1768.

Mertens, G., \& Morriss, J. (2021). Intolerance of uncertainty and threat reversal: A conceptual replication of Morriss et al. (2019). Behaviour Research and Therapy, 137, 103799.

Milad, M. R., \& Quirk, G. J. (2012). Fear extinction as a model for translational neuroscience: ten years of progress. Annual Review of Psychology, 63, 129-151.

Morriss, J., Bell, T., Johnstone, T., van Reekum, C. M., \& Hill, J. (2019a). Social domain based modulation of neural responses to threat: The different roles of romantic partners versus friends. Social Neuroscience, 14(4), 398-408.

Morriss, J., Bennett, K. P., \& Larson, C. L. (2021). I told you it was safe: Associations between intolerance of uncertainty and different parameters of uncertainty during instructed threat of shock. Journal of Behavior Therapy and Experimental Psychiatry, 70, 101620.

Morriss, J., Biagi, N., \& Dodd, H. (2020). Your guess is as good as mine: A registered report assessing physiological markers of fear and anxiety to the unknown in individuals with varying levels of intolerance of uncertainty. International Journal of Psychophysiology, 156, 93 104.

Morriss, J., Christakou, A., \& Van Reekum, C. M. (2015). Intolerance of uncertainty predicts fear extinction in amygdala-ventromedial prefrontal cortical circuitry. Biology of Mood \& Anxiety Disorders, $5(1), 1$.

Morriss, J., Christakou, A., \& Van Reekum, C. M. (2016). Nothing is safe: Intolerance of uncertainty is associated with compromised fear extinction learning. Biological Psychology, 121, 187-193.

Morriss, J., Gell, M., \& van Reekum, C. M. (2019b). The uncertain brain: A co-ordinate based meta-analysis of the neural signatures supporting uncertainty during different contexts. Neuroscience \& Biobehavioral Reviews, 96, 241-249.

Oglesby, M. E., Allan, N. P., \& Schmidt, N. B. (2017). Randomized control trial investigating the efficacy of a computer-based intolerance of uncertainty intervention. Behaviour Research and Therapy, 95, 50-57.

Peters, A., McEwen, B. S., \& Friston, K. (2017). Uncertainty and stress: Why it causes diseases and how it is mastered by the brain. Progress in Neurobiology, 156, 164-188.

Robichaud, M., \& Dugas, M. J. (2006). A cognitive-behavioral treatment targeting intolerance of uncertainty. In G.C. Davey \& A. Wells (Eds.), Worry and its psychological disorders: theory, assessment and treatment (pp. 289-304). Chichester: Wiley.

Salkovskis, P. M. (1996). The cognitive approach to anxiety: Threat beliefs, safety-seeking behavior, and the special case of health anxiety and obsessions. In P. M. Salkovskis (Ed.), Frontiers of cognitive therapy (pp. 48-74). New York: The Guilford Press.

Schienle, A., Köchel, A., Ebner, F., Reishofer, G., \& Schäfer, A. (2010). Neural correlates of intolerance of uncertainty. Neuroscience letters, 479(3), 272-276.

Shankman, S. A., Gorka, S. M., Nelson, B. D., Fitzgerald, D. A., Phan, K. L., \& O'Daly, O. (2014). Anterior insula responds to temporally unpredictable aversiveness: an fMRI study. Neuroreport, 25(8), 596.

Shihata, S., McEvoy, P. M., Mullan, B. A., \& Carleton, R. N. (2016). Intolerance of uncertainty in emotional disorders: What uncertainties remain? Journal of Anxiety Disorders, 41, 115-124.

Simmons, A., Matthews, S. C., Paulus, M. P., \& Stein, M. B. (2008). Intolerance of uncertainty correlates with insula activation during affective ambiguity. Neuroscience Letters, 430(2), 92-97.

Smith, S. M. (2002). Fast robust automated brain extraction. Human Brain Mapping, 17(3), 143-155.

Somerville, L. H., Wagner, D. D., Wig, G. S., Moran, J. M., Whalen, P. J., \& Kelley, W. M. (2013). Interactions between transient and 
sustained neural signals support the generation and regulation of anxious emotion. Cerebral Cortex, 23(1), 49-60.

Spielberger, C.D., Gorsuch, R.L., Lushene, R.E., Vagg, P.R. and Jacobs,

G. (1983). A Manual for the StateTrait Anxiety Inventory. Consulting Psychologists, Palo Alto, CA.

Tanovic, E., Gee, D. G., \& Joormann, J. (2018). Intolerance of uncertainty: Neural and psychophysiological correlates of the perception of uncertainty as threatening. Clinical Psychology Review, 60, 87-99.
Tashjian, S. M., Zbozinek, T. D., \& Mobbs, D. (2021). A Decision Architecture for Safety Computations. Trends in Cognitive Sciences, 25(5), 342-354.

Publisher's note Springer Nature remains neutral with regard to jurisdictional claims in published maps and institutional affiliations. 